\title{
The number of $F$-matchings in almost every tree is a zero residue
}

\author{
Noga Alon* \\ Sackler School of Mathematical Sciences \\ and Blavatnik School of Computer Science \\ Tel Aviv University
}

\author{
Simi Haber \\ Sackler School of Mathematical Sciences \\ Tel Aviv University \\ habbersi@post.tau.ac.il
}

\author{
Michael Krivelevich ${ }^{\dagger}$ \\ Sackler School of Mathematical Sciences \\ Tel Aviv University \\ krivelev@post.tau.ac.il.
}

Submitted: Jun 28, 2010; Accepted: Jan 27, 2011; Published: Feb 14, 2011

Mathematics Subject Classification: 05C30; 05A16, 05C05, 05C69, 92E10

\begin{abstract}
For graphs $F$ and $G$ an $F$-matching in $G$ is a subgraph of $G$ consisting of pairwise vertex disjoint copies of $F$. The number of $F$-matchings in $G$ is denoted by $s(F, G)$. We show that for every fixed positive integer $m$ and every fixed tree $F$, the probability that $s\left(F, \mathcal{T}_{n}\right) \equiv 0(\bmod m)$, where $\mathcal{T}_{n}$ is a random labeled tree with $n$ vertices, tends to one exponentially fast as $n$ grows to infinity. A similar result is proven for induced $F$-matchings. As a very special special case this implies that the number of independent sets in a random labeled tree is almost surely a zero residue. A recent result of Wagner shows that this is the case for random unlabeled trees as well.
\end{abstract}

\section{Introduction}

The number of independent sets in graphs is an important counting parameter. It is particularly well-studied for trees and tree-like structures. Prodinger and Tichy showed

\footnotetext{
* Research supported in part by an ERC advanced grant, by a USA-Israeli BSF grant and by the Hermann Minkowski Minerva Center for Geometry at Tel Aviv University.

${ }^{\dagger}$ Research supported in part by USA-Israel BSF Grant 2006322, by grant 1063/08 from the Israel Science Foundation, and by a Pazy memorial award.
} 
in [12] that the star and the path maximize and minimize, respectively, the number of independent sets among all trees of a given size. Part of the interest in this graph invariant stems from the fact that the number of independent sets plays a role in statistical physics as well as in mathematical chemistry, where it is known as the Merrifield-Simmons index [11]. A problem that arises in this context is the inverse problem: determine a graph within a given class of graphs (such as the class of all trees) with a given number of independent sets. It is an open conjecture [9] (see also [8]) that all but finitely many positive integers can be represented as the number of independent sets of some tree. Recently Wagner [14] published a surprising result that may partially explain why the inverse problem for independent sets in trees is difficult. He showed that for every positive integer $m$, the number of independent sets in a random tree with $n$ vertices is zero modulo $m$ with probability exponentially close to one. Wagner's proof does not give an intuitive explanation of the aforementioned fact. In this paper we give a probabilistic proof for the analogous result in labeled trees. Our proof is intuitive and simple, thus allowing us to generalize the result significantly. We refer the reader to [14] or [7] for further motivation and for a recent survey of previous results regarding the number of independent sets in trees.

Another graph parameter popular in statistical physics and in mathematical chemistry is the Hosoya index which is the number of matchings in the graph. While the inverse problem for the number of matchings in trees is easy, as the star with $n$ vertices has exactly $n$ matchings, finding the distribution of this number is still open, as is the case with the number of independent sets. Wagner mentions in [14] that his method could be applied to the number of matchings as well, showing that asymptotically this number is typically divisible by any constant $m$. This may serve as an explanation for the hardness of obtaining distribution results.

Both independent sets and matchings are special cases of $F$-matchings. Let $F$ and $G$ be graphs. An $F$-matching in $G$ is a subgraph of $G$ consisting of pairwise vertex disjoint copies of $F$. We say that the $F$-matching is induced in $G$ if no additional edge of $G$ is spanned by the vertices of $G$ covered by the matching. These two closely related notions generalize naturally matchings and independent sets. Indeed, if $F$ is the graph with two vertices and one edge then an $F$-matching is simply a matching. If $F$ is a single vertex then an induced $F$-matching is an independent set.

The notion of $F$-matching, which is sometimes called $F$-packing (and in the spanning case $F$-factor) has been considered in several papers, but most of them do not deal with the number of $F$-packings and mainly consider necessary or sufficient conditions for their existence. See, for example, [6] and its references.

Given graphs $F$ and $G$ we denote the set of $F$-matchings in $G$ by $S(F, G)$ and its size by $s(F, G)$. The set of all induced $F$-matchings in $G$ is denoted by $S^{\prime}(F, G)$ with $s^{\prime}(F, G)=\left|S^{\prime}(F, G)\right|$ being its size.

In this paper $G$ will be drawn at random from a probability space of graphs. We define the random tree $\mathcal{T}_{n}$ to be the set of all $n^{n-2}$ labeled trees on $n$ vertices endowed with the uniform distribution.

Our main results are the following: 
Theorem 1. Let $F$ be a tree that is not a single vertex and let $m$ be a positive integer. Then there is a constant $c=c(F, m)>0$ such that the number of $F$-matchings in the random tree $\mathcal{T}_{n}$ is zero modulo $m$ with probability at least $1-e^{-c n}$.

Note that when $F$ is a single vertex, the number of $F$-matchings in any graph with $n$ vertices is $2^{n}$.

Theorem 2. Let $F$ be a tree and let $m$ be a positive integer. Then there is a constant $c^{\prime}=c^{\prime}(F, m)>0$ such that the number of induced $F$-matchings in the random tree $\mathcal{T}_{n}$ is zero modulo $m$ with probability at least $1-e^{-c^{\prime} n}$.

The fact that the number of independent sets is almost surely a zero residue is an immediate consequence of Theorem 2 - simply take $F$ to be a single vertex.

In the next section we prove Theorem 1, in Section 3 we describe a similar proof of the induced case and in the last section we state some extensions and conclude with a few remarks and open questions. Our extensions include the fact that the assertions of both theorems hold when the random tree $\mathcal{T}_{n}$ is replaced by a random planar graph on $n$ vertices.

\section{The non-induced case}

In this section we prove Theorem 1. The proof is probabilistic and has two parts, a probabilistic claim (Lemma 3) and a deterministic claim (Lemma 5). Theorem 1 is an immediate consequence of these claims.

We shall use the following notation. Let $T$ be a tree and assume that $\{u, v\}$ is an edge in $T$. We define a rooted tree $T^{(u, v)}$ by first setting $v$ as the root — this defines a direction of parenthood in $T$ - and then removing $u$ along with its descendants. Note that $T^{(u, v)}$ is a rooted (undirected) tree. If $R$ is a rooted tree isomorphic to $T^{(u, v)}$ (a fact we denote by $R \cong T^{(u, v)}$ ) for some edge $\{u, v\} \in T$, we say that $T$ has an $R$-leaf. The next Lemma states that for every fixed rooted tree $R$, a random tree has an $R$-leaf with probability exponentially close to 1 .

Lemma 3. Let $R$ be a rooted tree. There exists a constant $c=c(R)>0$ such that

$$
\operatorname{Pr}\left[\exists\{u, v\} \in \mathcal{T}_{n} \text { s.t. } R \cong \mathcal{T}_{n}^{(u, v)}\right]>1-e^{-c n} .
$$

Proof. While our object of interest are trees, it is easier to work with functions on $[n]=$ $\{1,2, \ldots, n\}$ via the Joyal mapping ([5], also presented in English in [1]).

We shall briefly describe the Joyal mapping and some of its properties that we need. The Joyal mapping maps $f$, a function from $[n]$ to itself, to an undirected tree $T_{f}$ over the set of vertices $[n]$. There are $n^{n}$ functions in $[n]^{[n]}$, but only $n^{n-2}$ labeled trees over $[n]$. In order to make the mapping into a bijection we distinguish two vertices of a labeled tree by marking them left and right (we may mark one vertex with both). Now the target set is the set of all labeled trees over $[n]$ together with the markings, and is of size $n^{n}$. 
The mapping is defined as follows. Let $f:[n] \rightarrow[n]$. Define $\vec{G}_{f}$ as the functional digraph $^{1}$ with vertex set $[n]$ and edge set $\{(i, f(i)) \mid i \in[n]\}$. Every vertex in $\vec{G}_{f}$ has outdegree one, so every connected component has one directed cycle, and all edges that are not in a cycle are pointing towards the cycle. Let $M=\left\{a_{1}<a_{2}<\cdots<a_{m}\right\}$ be the set of all vertices participating in a cycle of $\vec{G}_{f}$. Notice that $M$ is the maximal set such that $\left.f\right|_{M}$ is a bijection. To get $T_{f}$, the tree corresponding to the function $f$, we first define a path by taking the vertices of $M$ and adding the $m-1$ edges of the form $\left\{f\left(a_{i}\right), f\left(a_{i+1}\right)\right\}$. We then mark $f\left(a_{1}\right)$ as "left" and $f\left(a_{m}\right)$ as "right". Finally we add the vertices in $[n] \backslash M$ with the edges $\{i, f(i)\}$ from $\vec{G}_{f}$ (forgetting about directions).

Given a tree $T$ with two such markings, we go back by defining $M$ as the vertices in the path $P$ connecting "left" and "right", and directing all other vertices towards $P$. Sort the members of $M$ according to their value and denote them by $a_{1}<a_{2}<\cdots<a_{m}$. We define $f$ as follows. If $i \in M$ is the $j$ 'th vertex in the path then $f(i)=a_{j}$. If $i \notin M$ then there is one edge, $(i, j)$, emanating from $i$, and we set $f(i)=j$. It is easy to verify that this is indeed the inverse of the mapping described above.

Notice that vertices that are not in a cycle are left by the Joyal mapping as they were in $\vec{G}_{f}$, meaning that they will be incident with exactly the same edges as in the functional graph. In particular, edges with both endpoints being vertices that are not in a cycle of $\vec{G}_{f}$ will touch the same edges in $T_{f}$ as in $\vec{G}_{f}$. For our purpose, the fate of vertices lying in a cycle is irrelevant.

Direct the edges of $R$ towards the root to get $\vec{R}$. Consider a random function $f$ on $[n]$ and let $X$ be the random variable counting the number of directed edges $(u, v)$ in $\vec{G}_{f}$ such that $u, v$ and the ancestors of $v$ in $\vec{G}_{f}$ do not belong to any cycle in $\vec{G}_{f}$, and in addition, $v$ and its ancestors form an isomorphic copy of $\vec{R}$.

Denote the vertices of $\vec{R}$ by $r_{1}, \ldots, r_{k}$, the root being $r_{k}$. Fix a $(k+1)$-tuple of vertices of $\vec{G}_{f}$, say $1,2, \ldots, k+1$. The probability that the edge $(k, k+1)$ meets the condition described above is at least the probability that $(k, k+1) \in E\left(\vec{G}_{f}\right)$, the mapping $i \rightarrow r_{i}$ is an isomorphism between $\vec{R}$ and $\vec{G}_{f}[\{1, \ldots, k\}]$, and in addition, there are no other edges of $\vec{G}_{f}$ incoming to $\{1, \ldots, k+1\}$. The latter is

$$
\left(\frac{1}{n}\right)^{k}\left(\frac{n-k-1}{n}\right)^{n-k}
$$

In order to see this simply notice that for $1 \leq i \leq k$ there is only one valid target for $f(i)$, while for $i \geq k+1$ it is enough to require that $f$ will map $i$ outside of $\{1,2, \ldots, k+1\}$. Therefore we get

$$
E X \geq\left(\begin{array}{c}
n \\
k+1
\end{array}\right) n^{-k}\left(\frac{n-k-1}{n}\right)^{n-k}
$$

which implies $E X=\Omega(n)$.

We want to show that $X$ is concentrated around its mean. Consider the value exposing martingale, in which we expose the values of $f$ one by one. Now, changing the value of $f$ in

\footnotetext{
${ }^{1} \mathrm{~A}$ functional digraph is a directed graph with all outdegrees equal one.
} 
one coordinate, $i$, can ruin at most two copies of $\vec{R}$ (one using the edge $(i, f(i))$ and another that now has an extra edge $\left.\left(i, f^{\prime}(i)\right)\right)$. Therefore the Lipschitz condition with constant two holds and we can apply the Azuma Inequality $[2,3]$ which yields $\operatorname{Pr}[X=0]<e^{-c n}$ for some constant $c>0$.

Observe that if $X(f)>0$ then by the definition of $X$, the corresponding tree $T_{f}$ contains the edge $\{u, v\}$ requested by the proposition.

As mentioned above, the Joyal correspondence is $n^{2}$ to one. If a labeled tree $T$ does not contain an edge as required, then all its $n^{2}$ preimages $f$ satisfy $X(f)=0$. Therefore, the probability not to get a tree with a required edge is at most $\operatorname{Pr}[X=0]<e^{-c n}$ as proven above.

Remark 4. Plugging the estimates in [2, Theorem 7.4.2] we can show that the constant $c$ above is bigger than $\left(100 k^{2 k+1}\right)^{-1}$.

The next argument of the proof states the existence of a nullifying tree $Z$ (depending on $F$ and $m)$ such that if a tree $T$ has a $Z$-leaf then $s(F, T) \equiv 0(\bmod m)$.

Lemma 5. Let $F$ be a tree with at least one edge and let $m$ be an integer. Then there exists a rooted tree $Z$ such that, if $Z \cong T^{(u, v)}$ for some edge $\{u, v\} \in T$, then $s(F, T) \equiv 0$ $(\bmod m)$.

Proof. The proof is constructive. By Proposition 6 to be proven below there exists a tree $Y$ such that $s(F, Y) \equiv 0(\bmod m)$.

Let $\Delta(F)$ be the maximal degree of $F$. To get $Z$ take $\Delta(F)+1$ copies of $Y$, add a new vertex $r$ to be viewed as the root of $Z$, and connect $r$ to a vertex of each $Y$ (thus adding $\Delta(F)+1$ edges).

Let $T$ be a tree and assume that $Z \cong T^{(u, v)}$ for some edge $\{u, v\} \in T$. We wish to show that $s(F, T) \equiv 0(\bmod m)$. There are finitely many ways in which one may cover $v$ by a copy of $F$, and it may also be that $v$ remains uncovered. We classify $F$-matchings in $T$ into classes $C_{1}, C_{2}, \ldots, C_{q}$ according to the copy of $F$ covering $v$, with the set of $F$-matchings not covering $v$ being a separate class $C_{0}$. We argue that the number of $F$-matchings in each such class is a zero residue. Indeed, the number of $F$-matchings in a given class $C_{i}$ is precisely the number of $F$-matchings in the forest remaining from $T$ after removing $v$ and the copy covering it, if there is one. In fact, this number is the product of the number of $F$-matchings in every connected component of the forest. By our construction of $Z$, at least one of the trees is this forest is isomorphic to $Y$. Since $s(F, Y) \equiv 0(\bmod m)$ we deduce that the number of $F$-matchings in the forest, and also in $C_{i}$, is zero modulo $m$. This is true for all $C_{i}$, and since $S(F, T)=\cup C_{i}$ one has $s(F, T) \equiv 0(\bmod m)$.

Before stating and proving the next proposition we define some notation. Let $F$ be a tree. Take a longest path in $F$ and denote its vertices by $u_{1}, u_{2}, \ldots, u_{l+1}$, where $l$ is the diameter of $F$. If we disconnect all edges of the form $\left\{u_{i}, u_{i+1}\right\}$ we get $l+1$ subtrees. Let $b_{i}$ be the number of vertices in the subtree containing $u_{i}$. With this notation we have $|F|=\sum_{i=1}^{l+1} b_{i}$. Since $b_{l+1}=1$ we may also write $|F|=1+\sum_{i=1}^{l} b_{i}$. We shall use this notation in the proof of the next proposition and in the proof of Proposition 9 as well. 
Proposition 6. Let $F$ be a tree with at least one edge and let $m$ be an integer. Then there exists a rooted tree $Y$ such that $s(F, Y) \equiv 0(\bmod m)$.

Proof. Let $W_{t}$ be a tree made of $t$ copies of $F$ in which we identify the vertex $u_{l+1}$ of copy $i$ with the vertex $u_{1}$ of copy $i+1$ (for $1 \leq i \leq t-1$ ). Let $P \subset W_{t}$ be the path in $W_{t}$ connecting the first copy of $u_{1}$ to the last copy of $u_{l+1}$, and number its vertices by $1, \ldots, l t+1$ in the natural order, from the copy of $u_{1}$ in the first copy of $F$ to the copy of $u_{l+1}$ in the last copy of $F$. We want to have a direction of parenthood in $W_{t}$, so we set 1 to be the root. Notice that all connected components of $W_{t} \backslash V[P]$ are of size strictly less than $|F|$.

We are interested in embeddings of $F$ in $W_{t}$, that is, in subgraphs of $W_{t}$ that are isomorphic to $F$. Notice that every such embedding must have a vertex in $P$. Let $C$ be an embedding of $F$ in $W_{t}$. We call the vertex $\min \{C \cap P\}$ the starting vertex of $C$. Consider the set of all starting vertices in $W_{t}$. If $1 \leq i \leq(t-2) l+1$ is a starting vertex, then by symmetry so is $i+l$. Observe that trivially 1 is a starting vertex (and so are $l+1,2 l+1, \ldots)$. By the symmetry argument above, if there are $d$ starting vertices between 1 and $l+1$ (inclusive), then there are $1+(t-1)(d-1)$ starting vertices in $W_{t}$. To see this recall that 1 is always a starting vertex, and each copy but the last adds $d-1$ starting vertices; also, the last copy of $F$ in $W_{t}$ does not contain any starting vertices apart from $1+l(t-1)$ as deleting $1+l(t-1)$ leaves less than $|F|$ vertices to the right of it. Similarly, if $i$ is a starting vertex then there are $d$ starting vertices between $i$ and $i+l$, inclusive.

Now we can define $\left\{Y_{r}\right\}$, a family of subtrees of $W_{t}$ a member of which will eventually be the sought after tree. Set $t$ to be large enough $(t=1+\lceil(r-1) /(d-1)\rceil$ will do $)$. To get $Y_{r}$ take the minimal subpath of $P \subset W_{t}$ containing the last $r$ starting vertices and then append to each vertex in the subpath the subtree of its descendants through children outside $P$. For example, $Y_{1}$ is the single starting vertex $1+l(t-1)$ and $Y_{d}$ is the next to the last copy of $F$ in $W_{t}$.

Let $g(r)$ be the number of $F$-matchings in $Y_{r}$. We count such $F$-matchings by the membership of $i$, the first vertex in $Y_{r}$. If $i$ is not covered by the matching, then the next embedding of $F$ begins no earlier than the next starting vertex. This means that the number of $F$-matchings of $Y_{r}$ in which $i$ is not covered is $g(r-1)$.

We argue now that if $i$ is covered by the matching then the next $d-1$ starting vertices are also covered. Let $\varphi: F \rightarrow Y_{r}$ be an embedding covering $i$. We claim that the next $d-1$ starting vertices are also covered by $\varphi$. First, since the diameter of $F$ is $l$, no vertex of $P$ farther than $i+l$ (which is the starting vertex $d-1$ away from $i$ ) is covered by $\varphi$. On the other hand, the path from $i$ to $i+l-1$ contains one copy of each $u_{i}$ (not necessarily in the natural order). Thus, the number of vertices in the set containing $i, i+1, \ldots, i+l-1$ and their descendants is exactly $\sum_{i=1}^{l} b_{i}$, hence $\varphi$ extends also to $i+l$. Therefore, the other embeddings in the $F$-matching need to start after $i+l$. We get that the number of such matchings is exactly $g(r-d)$. This gives the recursion $g(r)=g(r-1)+g(r-d)$.

Observe that the tree $Y_{r}, 1 \leq r<d$, does not contain a copy of $F$, and thus the only $F$-matching in $Y_{r}$ is the empty one, implying $g(r)=1$ for every $1 \leq r<d$; also, $g(d)=2$ as $Y_{d}=F$. We can extend the recursion backwards by defining $g(0)=1$ and $g(-1)=0$. By Claim 7 below there is an integer $r_{0}>0$ such that $g\left(r_{0}\right) \equiv 0(\bmod m)$. 
Define $Y=Y_{r_{0}}$. By the definition of $g(r)$ we have $s(F, Y) \equiv 0(\bmod m)$.

Claim 7. Let $g(r): \mathbb{N} \rightarrow \mathbb{Z}$ be a sequence of integers obeying a recurrence relation with integer coefficients $g(r)=\sum_{i=1}^{d} c_{i} g(r-i)$. Assume that $g(0)=0$ and $c_{d}=1$. Then for every positive integer $m>0$ there exists an index $r_{0}=r_{0}(m)>0$ such that $g\left(r_{0}\right) \equiv 0$ $(\bmod m)$.

Proof. First we claim that $g(r)(\bmod m)$ is periodic. Indeed, since $g(r)(\bmod m)$ is determined by the $d$-tuple of the previous $d$ values, and since modulo $m$ there are at most $m^{d}$ possible $d$-tuples, then after at most $m^{d}$ steps the sequence $g(r)(\bmod m)$ must become periodic. Next we claim that $g(r)(\bmod m)$ is periodic from the beginning. To see this simply extend the sequence $m^{d}$ steps backwards using the recurrence relation $g(r-d)=g(r)-\sum_{i=1}^{d-1} c_{i} g(r-i)$. The previous argument shows that the extended sequence is periodic starting at most at the $m^{d}$ 'th element, which is the first element of the original sequence. Hence $g(r)(\bmod m)$ is periodic from its first element, $g(0)=0$, and thus there is some $r_{0}>0$ such that $g\left(r_{0}\right) \equiv 0(\bmod m)$.

For more information on recurrence sequences modulo $m$, in particular for a better estimate of the index of the first zero residue element, see [4, Section 6.3].

\section{The induced case}

In this section we prove Theorem 2. The proof is similar to the proof of Theorem 1 and we shall focus on the differences between the proofs. As before, the proof is probabilistic. Lemma 3 is the probabilistic part here as well, but the deterministic part is replaced by Lemma 8 below.

We begin by constructing a nullifying rooted tree from copies of a tree $Y^{\prime}$ having $s^{\prime}\left(F, Y^{\prime}\right) \equiv 0(\bmod m)$.

Lemma 8. Let $F$ be a tree and let $m$ be an integer. There exists a rooted tree $Z^{\prime}$ such that if $Z^{\prime} \cong T^{(u, v)}$ for some edge $\{u, v\} \in T$, then $s^{\prime}(F, T) \equiv 0(\bmod m)$.

Proof. By Proposition 9 below there exists a tree $Y^{\prime}$ such that $s^{\prime}\left(F, Y^{\prime}\right) \equiv 0(\bmod m)$. Construct $Z^{\prime}$ by taking $\Delta(F)+2$ copies of $Y^{\prime}$, adding a new vertex $r$ to be viewed as the root of $Z$ ', connecting one copy to $r$ with a new edge and connecting the rest of the $\Delta(F)+1$ copies to $r$ via a path of length two.

Let $T$ be a tree and assume that $Z^{\prime} \cong T^{(u, v)}$ for some edge $\{u, v\} \in T$. We need to show that $s^{\prime}(F, T) \equiv 0(\bmod m)$.

There are finitely many ways in which $v$ may be covered by a copy of $F$, if it is covered at all. We classify induced $F$-matchings according to the copy of $F$ covering $v$. Denote these classes by $C_{1}, \ldots, C_{k}$ and let $C_{0}$ be the class of all induced $F$-matchings of $T$ in which $v$ is left uncovered. Clearly $S^{\prime}(F, T)=\bigcup_{i=0}^{k} C_{i}$. We claim that $\left|C_{i}\right| \equiv 0(\bmod m)$ for every $0 \leq i \leq k$.

Consider first the class $C_{0}$ of induced $F$-matchings in $T$ that leave $v$ uncovered. The number of such matchings is the number of matchings in the forest remaining after deleting 
$v$. This forest has a component isomorphic to $Y$ - the copy of $Y$ that was connected to $v$ by a single edge. The number of induced $F$-matchings in $C_{0}$ is then the product of the number of induced $F$-matchings in every connected component of the aforementioned forest which is zero modulo $m$.

Consider now the class $C_{i}$ for $i>0$. As before, there is a natural one to one correspondence between induced $F$-matchings in $T$ that belong to $C_{i}$ and induced $F$-matchings of the forest remaining after removing the copy of $F$ covering $v$ and all neighbors of vertices in that copy. Since $v$ is covered by the matching, all of its neighbors that are not covered by the same copy of $F$ must remain uncovered. Otherwise, an additional edge outside the copies of $F$ would be spanned. This means that in the above forest at least one of the $\Delta(F)+1$ copies that were connected to $v$ by a path of length two will now remain as a connected component. Hence, the number of induced $F$-matchings in $C_{i}$ is a zero residue.

Summing the sizes of the $C_{i}$ 's we get that $m^{\prime}(F, T) \equiv 0(\bmod m)$.

Proposition 9. Let $F$ be a tree and let $m$ be an integer. Then there exists a rooted tree $Y^{\prime}$ such that $s^{\prime}\left(F, Y^{\prime}\right) \equiv 0(\bmod m)$.

Proof. The construction and the proof are similar to those in the proof of Proposition 6, and we shall use the notation defined just before it. We define $W_{t}^{\prime}$ as a collection of $t$ disjoint copies of $F$, and we add an edge between the vertex $u_{l+1}$ of the $i$ 'th copy and the vertex $u_{1}$ of the $(i+1)^{\prime}$ 'th copy. We think of the first copy of $u_{1}$ as the root of $W_{t}^{\prime}$.

Let $P^{\prime}$ be the path connecting the first copy of $u_{1}$ with the last copy of $u_{l+1}$ and denote its vertices by $1, \ldots, t(l+1)$ in the natural order. We define starting vertices in the same manner as in the proof of Lemma 5 . The symmetry argument still holds, only now the period is $l+1$, that is, if $1 \leq i \leq(t-2)(l+1)+1$ is a starting vertex then so is $i+l+1$. Also, if there are $d$ starting vertices between 1 and $l+1$, then there are $d$ starting vertices between every starting vertex $i$ and $i+l$ and all in all there are $(t-1) d+1$ starting vertices in $W_{t}^{\prime}$.

Let $Y_{r}^{\prime}$ be the subgraph of $W_{t}^{\prime}$ composed of the minimal path of $P$ containing the last $r$ starting vertices together with their descendants through vertices that are not in $P$. Hence, $Y_{1}^{\prime}$ is a single vertex and $Y_{d+1}^{\prime}$ is a copy of $F$ with an extra vertex connected to $u_{l+1}$. Finally we define $g^{\prime}(r)$ as the number of induced $F$-matchings in $Y_{r}^{\prime}$.

We wish to derive a recurrence formula for $g^{\prime}(r)$. We count induced $F$-matchings of $Y_{r}^{\prime}$ by the membership of the first vertex. The number of induced $F$-matchings that do not cover the first vertex (who is also the first starting vertex) is exactly $g^{\prime}(r-1)$.

Consider matchings in which the first starting vertex $i$ is covered. The embedding of $F$ covering $i$ can not cover vertices of $P$ farther than $i+l$, since the diameter of $F$ is $l$. On the other hand, the number of vertices in the subgraph made of the path connecting $i$ to $i+l$ together with their descendants that are not in $P$ is exactly $\sum b_{i}=|F|$. Hence $i+l$ is also covered by the same embedding that covers $i$. Now, if $i+l+1$ is covered by another embedding of $F$, then $\{i+l, i+l+1\}$ is spanned, which is forbidden, so $i+l+1$ is not covered. Since there are $d$ starting vertices between $i$ and $i+l$, and since $i+l+1$ is a starting vertex as well, we get that the number of such matchings is exactly $g^{\prime}(r-d-1)$. Therefore we have $g^{\prime}(r)=g^{\prime}(r-1)+g^{\prime}(r-d-1)$. 
Clearly $g^{\prime}(r)=1$ for every $1 \leq r \leq d-1$, as the number of vertices in $Y_{r}^{\prime}$ in these cases is smaller than $|F|$. The value of $g^{\prime}(d)$ may be either 1 or 2 , depending on whether $F$ may be embedded into $Y_{d}$ or not. The value of $g^{\prime}(d+1)$ can also be one of a few options. Still, we extend $g^{\prime}$ backwards by defining $g^{\prime}(0)=g^{\prime}(d+1)-g^{\prime}(d), g^{\prime}(-1)=g^{\prime}(d)-g^{\prime}(d-1)$, and $g^{\prime}(-2)=g^{\prime}(d-1)-g^{\prime}(d-2)=0$. We complete the proof by applying Claim 7 .

\section{Concluding discussion}

Our initial objective was to provide a simple and intuitive explanation to the fact that almost all labeled trees have an even number of independent sets. Indeed, there are nullifying trees $Z$ such that when a tree $T$ has a $Z$-leaf, the number of independent sets in $T$ is even. Also, every fixed tree $Z$ appears as a $Z$-leaf in a random tree with $n$ vertices with probability tending to one as $n$ goes to infinity. Therefore almost all trees have an even number of independent sets.

The simplicity of the explanation allowed vast generalizations — Theorems 1 and 2 above. In fact, the proof also works in other scenarios. If a probability space of graphs has a property corresponding to the probabilistic part of the proof, then the number of (induced) $F$-matchings will be a zero residue in that probability space as well.

As a concrete example, let $\mathcal{P}_{n}$ be the random planar graph of order $n$, that is, $\mathcal{P}_{n}$ is the set of all simple labeled planar graphs with $n$ vertices endowed with the uniform distribution. In [10] it is shown that with probability exponentially close to one, $\mathcal{P}_{n}$ has an $R$-leaf for every fixed rooted tree $R$. Thus, by the above, the number of (induced) $F$-matchings is a zero residue in a random planar graph. Notice that $\mathcal{P}_{n}$ is connected with probability at least $1 / e$ as shown in [10], so a potential simpler strategy of proving the same result - showing the existence of a component having a zero residue number of (induced) $F$-matchings — will not suffice.

Similar results may be obtained for other random graphs models as well. On the other hand, if we consider dense random graphs then a different approach is required. For example, it is not clear how the number of independent sets typically behaves as a residue for the binomial random graph $G(n, 1 / 2)$. Moreover, it is not difficult to show that for $p=p(n)$ close to 1 in the range in which the maximum independent set of $G(n, p)$ is $\Theta(1)>1$ asymptotically almost surely, the number of independent sets in $G(n, p)$ is nearly uniformly distributed modulo any constant $m$. See [13] for several related results.

Our proof implies that the number of $F$-matchings in a random tree of order $n$ is typically zero modulo any constant $m$ when the size of $F$ grows slowly enough with $n$. It may be interesting to find the maximal rate of growth for which this property still holds.

In a recent paper [7] Law shows that for every two integers $k, m$, there exists a tree $T$ such that the number of independent sets of $T$ is congruent to $k$ modulo $m$. One may ask if the same holds for general (induced) $F$-matchings as well. Is it true that for every tree $F$ and integers $k, m$ there exists a tree $T$ such that the number of (induced) $F$-matchings of $T$ is congruent to $k$ modulo $m$ ?

Using Remark 4 we can estimate the constants appearing in Theorems 1 and 2. Clearly the constant will depend on the size of the nullifying tree. No effort was made to find the 
smallest nullifying tree.

Acknowledgment We thank Alan Frieze for suggesting the use of the Joyal Correspondence in the proof of Lemma 3.

\section{References}

[1] M. Aigner and G. M. Ziegler. Proofs from THE BOOK. Springer-Verlag, Berlin, third edition, Nov. 2003.

[2] N. Alon and J. H. Spencer. The Probabilistic Method. Wiley-Interscience Series in Discrete Mathematics and Optimization. Wiley, New Jersey, Third edition, Aug. 2008.

[3] K. Azuma. Weighted sums of certain dependent random variables. Tôhoku Mathematical Journal, 19(3):357-367, 1967.

[4] G. Everest, A. van der Poorten, I. Shparlinski, and T. Ward. Recurrence Sequences. Number 104 in Mathematical Surveys and Monographs. American Mathematical Society, USA, Aug. 2003.

[5] A. Joyal. Une théorie combinatoire des séries formelles. Advances in Mathematics, 42(1):1-82, Oct. 1981.

[6] D. Kühn and D. Osthus. Embedding large subgraphs into dense graphs. Surveys in combinatorics 2009, 137-167, London Math. Soc. Lecture Note Ser., 365, Cambridge Univ. Press, Cambridge, 2009.

[7] H.-F. Law. On the number of independent sets in a tree. Electronic Journal of Combinatorics, 17, 2010. 5pp, N18.

[8] X. Li, Z. Li, and L. Wang. The inverse problems for some topological indices in combinatorial chemistry. Journal of Computational Biology, 10(1):47-55, Feb. 2003.

[9] V. Linek. Bipartite graphs can have any number of independent sets. Discrete Mathematics, 76(2):131-136, June 1989.

[10] C. McDiarmid, A. Steger, and D. J. Welsh. Random planar graphs. Journal of Combinatorial Theory, Series B, 93:187-205, 2005.

[11] R. E. Merrifield and H. E. Simmons. Topological Methods In Chemistry. John Wiley \& Sons, Inc., New York, Mar. 1989.

[12] H. Prodinger and R. F. Tichy. Fibonacci numbers of graphs. The Fibonacci Quarterly, 20(1):16-21, 1982.

[13] A. Scott and A. Tateno. On the number of triangles and other complete graphs in a random graph. In preparation, 2010.

[14] S. G. Wagner. Almost all trees have an even number of independent sets. The Electronic Journal of Combinatorics, 16(1), July 2009. \#R93. 